\title{
Cultural Terms Translation in Japan-Indonesia Diplomacy Content on Social Media Instagram
}

\author{
Asteria Permata Martawijaya ${ }^{1, *}$, Syihabuddin $^{2}$, Dadang Sudana ${ }^{2}$, Dedi Sutedi ${ }^{2}$ \\ ${ }^{1}$ STBA YAPARI-ABA Bandung, Universitas Pendidikan Indonesia, Bandung, Indonesia \\ ${ }^{2}$ Linguistics Study Program, Universitas Pendidikan Indonesia, Bandung, Indonesia \\ *Corresponding author.email: asteria@stba.ac.id
}

\begin{abstract}
On current era of digitalization information, the Japanese Embassy for Indonesia is developing its diplomacy by using several social media platforms including Instagram as a means of cultural diplomacy. Japan cultural diplomacy in social media Instagram carried out in two languages. The Japanese content is written along with the Indonesian through a translation process. This makes translation become a form of Japanese cultural diplomacy in social media. Related to the topic of this research, the translation of cultural diplomacy content on social media must be done in such a way that the translation results used in the content can support the cultural diplomacy function. The translation of cultural diplomacy content delivered through the Instagram platform allows the use of different translation techniques than with translation in other media. The problem studied in this study is about the technique of translating cultural terms on Japan Embassy for Indonesia's social media Instagram content. The source of research data is in the form of content uploaded by the Japanese Ambassador to Indonesia, Kanasugi Kenji on Instagram social media in the early period of his tenure as the Japanese Ambassador for Indonesia from January 2021 to May 2021. Research data obtained by using the note method and analyzed using qualitative data analysis methods. From the results of the study, it was obtained an overview of the translation techniques used in translating cultural terms in Japanese-Indonesian diplomatic content on social media Instagram in its function as a means of conveying diplomacy in the digital world which is expected to provide evaluation material and input regarding translation technique in the diplomatic field and the digital world.
\end{abstract}

Keywords: translation techniques, cultural terms, japan diplomacy, Instagram.

\section{INTRODUCTION}

In the current era of digitalization information, Japan is developing a form of diplomacy by using digital diplomacy as one of their public diplomacy media. Especially in Indonesia, the Japan Embassy for Indonesia is developing its digital diplomacy by using several social media platforms including Instagram as a means of its country's cultural diplomacy. In this case, the Ambassador of Japan acts as a social media influencer who shares content related to cultural diplomacy in the content on his social media.

The interesting thing about Japan's cultural diplomacy in Instagram is that the Japan embassy for Indonesia uses two languages in the uploaded content. The Japanese content is written along with the Indonesian content by means of a translation process. This indirectly makes translation a form of Japanese cultural diplomacy in social media.

Translation is a communication tool. As a communication tool, translation has a communicative purpose set by the author of the source language text, the translator as a mediator, and the reader of the target language text. The goal setting is strongly influenced by the social and cultural context as well as the ideology of the source language text writer, translator, and the client or target language text reader. In many cases, the culture of the writer of the source language text is very different from the culture of the reader of the target language text, and so are the ideologies of the translator and the client which are often different from one another. Because the translation is aimed at the readers of the target language text, the translator's attention must be directed to finding 
an equivalent according to the culture of the target language text reader (Fahrurrozi \& Wicaksono, 2016).

The accuracy of the transfer of messages is used as one of the important aspects of a quality translation, not without reason. If we review some of the definitions put forward by translator experts, the problem of message transfer is more emphasized than the transfer of language forms. As stated by Catford (1965: 20) that translation is "The replacement of textual material in one language (SL) by equivalent textual material in another language (TL)". This opinion is confirmed by Nida \& Taber (in Shuttleworth \& Cowie, 1997: 182) which states "Translating consists in reproducing in the receptor language the closest natural equivalent of the sourcelanguage message, first in terms of meaning and secondly in terms of style. " In fact, there is agreement among experts that a text is called a translation if the text has an equivalent relationship with the source language text.

When translating, a translator carries out planned activities aimed at bridging two different cultural spheres. The translated text is the embodiment of the procedures, rules and principles that are the general conventions of writing certain types of discourse. The convention is reflected in the choice of lexical vocabulary and grammatical patterns that characterize a certain type of reality. Certain linguistic tools become a regular feature (text identity) of the construction of a discourse. The textual identity is theoretically a reflection of linguistic conversion but also a reflection of issues related to politics, socio-culture, gender, age and others (Machali, 2013: 33).

The thing to explore is how do translators translate cultural diplomacy content in Instagram social media? Therefore, it is interesting to study what techniques are used by translators in translating cultural terms in diplomatic content through Instagram social media.

The research questions that form the basis of this research are as follows: "what translation techniques are used in translating Japanese-Indonesian cultural diplomacy content on Instagram social media?". This research question will be answered through the following research problem formulations: 1) What cultural terms are used in the cultural diplomacy content uploaded by the Japanese Embassy to Indonesia on Instagram? and 2) What translation techniques are used in translating cultural terms for cultural diplomacy content uploaded by the Japanese Embassy to Indonesia on Instagram?
This study aims to describe the techniques used by translators when translating cultural terms in cultural diplomacy content uploaded on social media Instagram. The classification of cultural terms used in this study is classification theory from Newmark (1988:95) which classifies cultural terms into 5 categories: 1) ecology, 2) material culture (artefacts), 3) social culture, 4) organizations, customs, activities, procedures, concepts, and 5) Gestures and habits. Regarding the translation technique, this research is using Molina \& Albir (2002: 509-511) translation technique classification theory: adaptation, establish equivalent, amplification, borrowing, calque, compensation, description, discursive creation, establish equivalent, generalization, linguistic compression, literal translation, modulation, particularization, reduction, substitution, transposition and variations

\section{METHOD}

This study uses a qualitative descriptive research design in the form of content analysis as a research foundation. In qualitative research, content analysis emphasizes how researchers see the meaning of communication content, read symbols, and the meaning of the contents symbolic interactions that occur in communication (Bungin, 2008).

The data source used in this research is social media Instagram. In this study, there are two pieces of data, namely: (1) diplomacy content data in Japanese and (2) translation of diplomacy related uploads. The two data were uploaded by the Japan Embassy for Indonesia in social media Instagram. The selected uploads are 30 content uploaded by the Japan Ambassador to Indonesia, Kanasugi Kenji in the first period of his tenure as Japan Ambassador for Indonesia on January 2021-July 2021. 30 selected content based on the number of likes obtained above 10,000 likes from followers of the Japanese Embassy's Instagram account for Indonesia.

The data in this study are sentences containing words or phrases that have elements of cultural words collected by applying the note-taking method to document the uploads of the Japanese Embassy to Indonesia on Instagram social media. The data is obtained from words or phrases that contain cultural elements contained in $\mathrm{TSu}$, uploaded by the Japanese Embassy to Indonesia on Instagram social media. The data of this study were collected by reading and note-taking technique. The data 
were analyzed using qualitative data analysis which according to Huberman and Miles (2014: 31-33) consists of 3 steps of activities; data condensation, data display and conclusion drawing/verification. The data that has been collected is classified based on the category of cultural terms theory from Newmark (1988) and translation techniques theory from Molina \& Albir (2002). By using this analysis, it is hoped that the use of translation techniques in translating content on social media in its function as a means of conveying diplomacy in the digital world can be expressed more thoroughly and especially from a linguistic point of view.

\section{RESULT AND DISCUSSION}

\subsection{Result}

3.1.1 Cultural terms used in the diplomatic content of the Japanese Embassy to Indonesia on social media Instagram

This section contains a description of the research findings on the types of cultural terms in Japan diplomatic content on social media Instagram.

There are 42 cultural terms data that are used in the diplomatic content of the Japan Embassy for Indonesia on social media Instagram. Social Culture is the most widely used category that is used in 20 data or approximately $47,61 \%$ of the total data. The next category that is also widely used is material culture, that is used in 15 data or approximately $35.71 \%$ of the total data. Organizations, Customs and Idea Category used in 5 data or $11.90 \%$ of the total data and ecology category is used in 2 data or $4.76 \%$ of the total data obtained.

The social culture category is manifested in 5 data of Work type, 4 data of Time type, 6 data of Kinship type, 4 data of Honorific type and 1 data of activities type. Meanwhile, for the material culture category is manifested in 3 data of clothes type, 10 data of food type, 1 data of decoration type and 1 data ofsupplementary data. The categories of organizations, customs and ideas consist of 2 data of Government Position type, and 1 data of Event type. Administrative and Organization Furthermore, the ecology category only consists of 2 data on cultural terms with fauna type. For more details, see the table below.
Table 1. Cultural terms used in the diplomatic content of the Japanese Embassy to Indonesia on social media Instagram

\begin{tabular}{|c|c|c|c|c|c|}
\hline No & $\begin{array}{c}\text { Cultural } \\
\text { Terms } \\
\end{array}$ & Type & $\begin{array}{l}\text { Frequ } \\
\text { ency }\end{array}$ & \begin{tabular}{|c|} 
Total \\
Frequency \\
\end{tabular} & \begin{tabular}{|c|}
$\begin{array}{c}\text { Percenta } \\
\text { ge }\end{array}$ \\
\end{tabular} \\
\hline 1 & Ecology & Fauna & 2 & 2 & $4.76 \%$ \\
\hline \multirow[t]{4}{*}{2} & \multirow{4}{*}{$\begin{array}{l}\text { Material } \\
\text { Culture }\end{array}$} & Clothes & 3 & \multirow[t]{4}{*}{15} & \multirow{4}{*}{$35.71 \%$} \\
\hline & & Food & 10 & & \\
\hline & & Decorations & 1 & & \\
\hline & & Supplements & 1 & & \\
\hline \multirow[t]{5}{*}{3} & \multirow{5}{*}{$\begin{array}{l}\text { Social } \\
\text { Culture }\end{array}$} & Work & 5 & \multirow[t]{5}{*}{20} & \multirow[t]{5}{*}{$47.61 \%$} \\
\hline & & Time & 4 & & \\
\hline & & Kinship & 6 & & \\
\hline & & Honorific & 4 & & \\
\hline & & Activities & 1 & & \\
\hline \multirow[t]{4}{*}{4} & \multirow{4}{*}{$\begin{array}{l}\text { Organizat } \\
\text { ions, } \\
\text { Customs } \\
\text { and Idea }\end{array}$} & $\begin{array}{l}\text { Government } \\
\text { Position }\end{array}$ & 2 & \multirow[t]{4}{*}{5} & \multirow[t]{4}{*}{$11.90 \%$} \\
\hline & & Event & 1 & & \\
\hline & & Administrative & 1 & & \\
\hline & & Organization & 1 & & \\
\hline & Total & & & 42 & $100 \%$ \\
\hline
\end{tabular}

Some representative forms of cultural terms found in this study will be discussed as follows.

\section{Ecology}

Ecology is a type of cultural terms related to the geographical conditions that exist in the environment around community groups, for example: flora, fauna, valleys, hills and so on. From the 42 cultural terms data obtained, there are 2 data fauna type of ecological culture, as follows.

Table 2. The Data of Ecological Culture Terms Used in Diplomatic Content of the Japan Embassy for Indonesia on Social Media Instagram

\begin{tabular}{|l|l|l|l|l|}
\hline No & \multicolumn{1}{|c|}{ Tsu } & \multicolumn{1}{c|}{ Tsa } & Types & $\begin{array}{c}\text { Techni } \\
\text { que }\end{array}$ \\
\hline 1 & の (8) & $\begin{array}{l}\text { ikan cupang } \\
\text { atau Beta } \\
\text { dalam bahasa } \\
\text { Jepang. }\end{array}$ & fauna & $\begin{array}{l}\text { amplifi } \\
\text { kasi }\end{array}$ \\
\hline 2 & $(10)$ & ikan cupang & fauna & $\begin{array}{l}\text { reducti } \\
\text { on }\end{array}$ \\
\hline
\end{tabular}

The cultural terms used in the two data are actually the same, "nettaigyo no beta", but because the translation techniques used to translate these cultural terms are different, the two data are considered as two different data.

In data (1) nettai gyo no beta is translated by providing an explanation of a term (amplification technique) which is translated to "ikan cupang atau Beta dalam bahasa Jepang". While in data (2) nettai gyo no beta is translated by reducing/eliminating (reduction technique) to " ikan cupang ". 


\section{Material Culture}

Material culture consists of food, transportation, clothing, buildings, houses, vehicles or goods that are used daily. This category is closely related to the system of production, use and maintenance of living equipment and technology in a society. From the 42 cultural terms data obtained there are 15 material culture data, as follows.

Table 3. Data on Material Culture Terms Used in Diplomatic Content of the Japanese Embassy to Indonesia on Social Media Instagram

\begin{tabular}{|c|c|c|c|c|}
\hline No & $\mathrm{TSu}$ & $\mathrm{TSa}$ & Types & $\begin{array}{c}\text { Techniq } \\
\text { ue }\end{array}$ \\
\hline 1 & (4) & kimono & clotheses & $\begin{array}{l}\text { pure } \\
\text { borrowin } \\
\mathrm{g}\end{array}$ \\
\hline 2 & インドネシアの (6) & kebaya & clotheses & $\begin{array}{l}\text { establish } \\
\text { equivale } \\
\text { nt- } \\
\text { reductio } \\
\text { n }\end{array}$ \\
\hline 3 & (12) & batik & clotheses & $\begin{array}{l}\text { establish } \\
\text { equivale } \\
\text { nt }\end{array}$ \\
\hline 4 & $-\cdot(7)$ & $\begin{array}{l}\text { Mie } \\
\text { Ayam }\end{array}$ & food & $\begin{array}{l}\text { establish } \\
\text { equivale } \\
\text { nt }\end{array}$ \\
\hline 5 & () & bakso & food & $\begin{array}{l}\text { establish } \\
\text { equivale } \\
\text { nt- } \\
\text { reductio } \\
\text { n }\end{array}$ \\
\hline 6 & のつたー・(9) & $\begin{array}{l}\text { Mie } \\
\text { Kangkung }\end{array}$ & food & $\begin{array}{l}\text { establish } \\
\text { equivale } \\
\text { nt- } \\
\text { reductio } \\
\text { n }\end{array}$ \\
\hline 7 & (21) & $\begin{array}{l}\text { bumbu- } \\
\text { bumbu } \\
\text { spicy }\end{array}$ & food & calque \\
\hline 8 & $\begin{array}{l}\text { •（きしめんののきそば } \\
\text { ) }(13)\end{array}$ & $\begin{array}{l}\text { kwetiau } \\
\text { goreng }\end{array}$ & food & $\begin{array}{l}\text { establish } \\
\text { equivale } \\
\text { nt }\end{array}$ \\
\hline 9 & $-\cdot(14)$ & $\begin{array}{l}\text { Bihun } \\
\text { Goreng }\end{array}$ & food & $\begin{array}{l}\text { establish } \\
\text { equivale } \\
\text { nt }\end{array}$ \\
\hline 10 & （=のような） (18) & claypot & food & $\begin{array}{l}\text { pure } \\
\text { borrowin } \\
\text { g- } \\
\text { reductio } \\
\text { n }\end{array}$ \\
\hline
\end{tabular}

\begin{tabular}{|c|l|l|l|l|}
\hline 11 & きなげ (19) & $\begin{array}{l}\text { pangsit } \\
\text { goreng } \\
\text { yang } \\
\text { sangat } \\
\text { besar }\end{array}$ & food & $\begin{array}{l}\text { transposi } \\
\text { tion }\end{array}$ \\
\hline 12 & リの (19) & $\begin{array}{l}\text { mie } \\
\text { pangsit }\end{array}$ & food & $\begin{array}{l}\text { establish } \\
\text { equivale } \\
\text { nt }\end{array}$ \\
\hline 13 & $($ (の) (21) & "rendang" & food & $\begin{array}{l}\text { reductio } \\
\mathrm{n}\end{array}$ \\
\hline 14 & $(12)$ & $\begin{array}{l}\text { boneka } \\
\text { Hina }\end{array}$ & hiasan & $\begin{array}{l}\text { transposi } \\
\text { si }\end{array}$ \\
\hline 15 & $(23)$ & $\begin{array}{l}\text { vaksin } \\
\text { buatan } \\
\text { Astra } \\
\text { Zeneca }\end{array}$ & $\begin{array}{l}\text { supleme } \\
\mathrm{n}\end{array}$ & $\begin{array}{l}\text { transposi } \\
\text { tion }\end{array}$ \\
\hline
\end{tabular}

The social material category of Cultural terms that are used in the diplomatic content of the Japan Embassy from Indonesia are divided into types of clothing, decoration, food and supplements.

In the Japan diplomacy content on Instagram, cultural terms are used in the social material category that is absorption language from Indonesian. Therefore, in the translation process, the translator uses a lot of established equivalent and reduction techniques, using terms that are more familiar to the reader and eliminating part of the source text in the form of explanations. additional terms used.

\section{Social Culture}

Social culture category includes work, kinship, use of leisure, sports, games, entertainment, kinship terms and art. From the 42 cultural terms data obtained, there are 21 social culture data, which is divided into work, time, kinship and calling types, as follows.

Table 4. Data on social culture terms used in the diplomatic content of the Japanese embassy to Indonesia on Instagram social media

\begin{tabular}{|c|c|c|c|c|}
\hline $\mathrm{NO}$ & $\mathrm{TSu}$ & $\mathrm{TSa}$ & Jenis & $\begin{array}{c}\text { Techniq } \\
\text { ue }\end{array}$ \\
\hline 1 & でー (8) & $\begin{array}{l}\text { Di masa } \\
\text { pandemi } \\
\text { dan di- } \\
\text { rumah-aja } \\
\text { (stay- } \\
\text { home) }\end{array}$ & waktu & $\begin{array}{l}\text { particula } \\
\text { rization }\end{array}$ \\
\hline 2 & の(2) & $\begin{array}{l}\text { ketika } \\
\text { duduk di } \\
\text { bangku } \\
\text { kuliah }\end{array}$ & waktu & $\begin{array}{l}\text { establish } \\
\text { equivale } \\
\text { nt }\end{array}$ \\
\hline
\end{tabular}




\begin{tabular}{|c|c|c|c|c|}
\hline 3 & (3) & $\begin{array}{l}\text { masa } \\
\text { isolasi } \\
\text { mandiri }\end{array}$ & waktu & $\begin{array}{l}\text { establish } \\
\text { equivale } \\
\mathrm{nt}\end{array}$ \\
\hline 4 & (20) & $\begin{array}{l}\text { jaman } \\
\text { Orde Baru }\end{array}$ & waktu & $\begin{array}{l}\text { establish } \\
\text { equivale } \\
\text { nt }\end{array}$ \\
\hline 5 & (24) & $\begin{array}{l}\text { acara } \\
\text { penyeraha } \\
\text { n vaksin } \\
\text { secara } \\
\text { daring }\end{array}$ & activities & $\begin{array}{l}\text { transposi } \\
\text { si }\end{array}$ \\
\hline 6 & (2) & Madame & kinship & $\begin{array}{l}\text { establish } \\
\text { equivale } \\
\mathrm{nt}\end{array}$ \\
\hline 7 & とも(2) & Mereka & kinship & $\begin{array}{l}\text { generaliz } \\
\text { ation }\end{array}$ \\
\hline 8 & のたち (5) & $\begin{array}{l}\text { kedua } \\
\text { anak saya, } \\
\text { kebetulan } \\
\text { dua- } \\
\text { duanya } \\
\text { perempua } \\
\text { n. }\end{array}$ & kinship & $\begin{array}{l}\text { modulati } \\
\text { on }\end{array}$ \\
\hline 9 & (5) & $\begin{array}{l}\text { Yang } \\
\text { tertua }\end{array}$ & kinship & $\begin{array}{l}\text { generaliz } \\
\text { ation }\end{array}$ \\
\hline 10 & (5) & $\begin{array}{l}\text { anak } \\
\text { kedua } \\
\text { saya } \\
\end{array}$ & kinship & $\begin{array}{l}\text { particula } \\
\text { rization }\end{array}$ \\
\hline 11 & のの(22) & $\begin{array}{l}\text { cucu } \\
\text { pertama } \\
\text { saya dan } \\
\text { dia laki- } \\
\text { laki }\end{array}$ & kinship & $\begin{array}{l}\text { transposi } \\
\text { si }\end{array}$ \\
\hline 12 & (5) & $\begin{array}{l}\text { teman- } \\
\text { teman } \\
\text { semua. }\end{array}$ & $\begin{array}{l}\text { panggila } \\
\mathrm{n}\end{array}$ & $\begin{array}{l}\text { particula } \\
\text { rization }\end{array}$ \\
\hline 13 & (8) & $\begin{array}{l}\text { sahabat } \\
\text { IG }\end{array}$ & $\begin{array}{l}\text { Panggila } \\
\mathrm{n}\end{array}$ & $\begin{array}{l}\text { particula } \\
\text { rization }\end{array}$ \\
\hline 14 & (9) & $\begin{array}{l}\text { teman } \\
\text { semua }\end{array}$ & $\begin{array}{l}\text { Panggila } \\
\mathrm{n}\end{array}$ & $\begin{array}{l}\text { particula } \\
\text { rization }\end{array}$ \\
\hline 15 & (10) & $\begin{array}{l}\text { teman } \\
\text { semua }\end{array}$ & $\begin{array}{l}\text { Panggila } \\
\mathrm{n}\end{array}$ & $\begin{array}{l}\text { particula } \\
\text { rization }\end{array}$ \\
\hline 16 & さん(11) & $\begin{array}{l}\text { teman- } \\
\text { teman } \\
\text { instagram }\end{array}$ & $\begin{array}{l}\text { Panggila } \\
\mathrm{n}\end{array}$ & $\begin{array}{l}\text { particula } \\
\text { rization }\end{array}$ \\
\hline 17 & (8) & off & work & Calque \\
\hline 18 & をしたがあります。(5) & $\begin{array}{l}\text { membantu } \\
\text { kelas } \\
\text { bahasa } \\
\text { Jepang. }\end{array}$ & work & $\begin{array}{l}\text { establish } \\
\text { equivale } \\
\text { nt }\end{array}$ \\
\hline 19 & $-(24)$ & $\begin{array}{l}\text { \#diRumah } \\
\underline{\text { Aja }}\end{array}$ & work & $\begin{array}{l}\text { establish } \\
\text { equivale } \\
\text { nt }\end{array}$ \\
\hline
\end{tabular}

\begin{tabular}{|l|l|l|l|l|}
\hline 20 & $-(25)$ & WFH & work & $\begin{array}{l}\text { establish } \\
\text { equivale } \\
\mathrm{nt}\end{array}$ \\
\hline 21 & $(2)$ & volunteer & work & $\begin{array}{l}\text { establish } \\
\text { equivale } \\
\mathrm{nt}\end{array}$ \\
\hline
\end{tabular}

The translation of cultural terms in the social culture category in the diplomatic content of the Japan embassy uses establish equivalent techniques and particularization techniques. The establish equivalent technique is widely used in the work types. While the particularization technique is used to translate the honorific types of cultural terms of this category.

\section{Organization, Customs and Idea}

The organizational culture, customs and ideas category of cultural terms including politics, administration, religion, art, historical concepts, street names, naming positions in government, organization names, and organizational procedures. From 42 data on cultural terms obtained, there are 5 data of this category, which is divided into the type of administration, position in government and organization, as follows.

Table 5. Data on the terms Organization, Customs and Idea used in the diplomatic content of the Japanese Embassy to Indonesia on Instagram social media

\begin{tabular}{|c|c|c|c|c|}
\hline $\mathrm{NO}$ & $\mathrm{TSu}$ & $\mathrm{TSa}$ & Jenis & $\begin{array}{l}\text { Techniq } \\
\text { ue }\end{array}$ \\
\hline 1 & () (16) & $\begin{array}{l}\text { Jabodetab } \\
\text { ek }\end{array}$ & $\begin{array}{l}\text { administ } \\
\text { ras }\end{array}$ & $\begin{array}{l}\text { establish } \\
\text { equivale } \\
\text { nt - } \\
\text { reductio } \\
\text { n }\end{array}$ \\
\hline 2 & りの(12) & $\begin{array}{l}\text { Hina- } \\
\text { Matsuri } \\
\text { (dolls } \\
\text { festival) } \\
\end{array}$ & event & $\begin{array}{l}\text { amplific } \\
\text { ation }\end{array}$ \\
\hline 3 & の(15) & $\begin{array}{l}\text { Yayasan } \\
\text { Warga } \\
\text { Persahaba } \\
\text { tan } \\
\text { (Fukushi } \\
\text { Tomo No } \\
\text { Kai) }\end{array}$ & $\begin{array}{l}\text { organiza } \\
\text { tion }\end{array}$ & $\begin{array}{l}\text { Literal } \\
\text { transatio } \\
\text { ns }\end{array}$ \\
\hline 4 & (6) & $\begin{array}{l}\text { Wakil } \\
\text { Ketua } \\
\text { DPR }\end{array}$ & $\begin{array}{l}\text { posisi } \\
\text { dalam } \\
\text { pemerint } \\
\text { ahan }\end{array}$ & $\begin{array}{l}\text { establish } \\
\text { equivale } \\
\text { nt }\end{array}$ \\
\hline 5 & ジヨコ大統領(22) & $\begin{array}{l}\text { Bapak } \\
\text { Presiden } \\
\text { Joko } \\
\text { Widodo }\end{array}$ & $\begin{array}{l}\text { posisi } \\
\text { pemerint } \\
\text { ahan }\end{array}$ & $\begin{array}{l}\text { establish } \\
\text { equivale } \\
\text { nt }\end{array}$ \\
\hline
\end{tabular}


As in the previous category, terms derived from Indonesian culture in this category are translated using the establish equivalent technique on the translation process. As for cultural terms that is originating from Japanese culture, the translator uses amplification techniques and literal translation techniques.

\subsubsection{The Translation Technique Used In} Translating Cultural Terms In The Diplomatic Content Of The Japanese Embassy To Indonesia On Social Media Instagram

This section contains a description of the research findings on the types of translation technique used on Japan diplomatic content on social media Instagram. For more details, see the table below.

Table 6. Translation Techniques and Amount of Data Usage

\begin{tabular}{|c|l|l|l|}
\hline No & \multicolumn{1}{|c|}{ Techique } & Frequency & Percentage \\
\hline $\mathbf{1}$ & Amplification & 2 & $4.25 \%$ \\
\hline $\mathbf{2}$ & Reduction & 6 & $12.76 \%$ \\
\hline $\mathbf{3}$ & Establish equivalent & 19 & $40.42 \%$ \\
\hline $\mathbf{4}$ & Pure Borrowing & 2 & $4.25 \%$ \\
\hline $\mathbf{5}$ & Transposition & 5 & $10.63 \%$ \\
\hline $\mathbf{6}$ & Calque & 3 & $6.38 \%$ \\
\hline $\mathbf{7}$ & Partiicularization & 7 & $14.89 \%$ \\
\hline $\mathbf{8}$ & Generalization & 2 & $4.25 \%$ \\
\hline $\mathbf{9}$ & Literal Translation & 1 & $2.12 \%$ \\
\hline & TOTAL & 47 & $100 \%$ \\
\hline
\end{tabular}

\section{Amplification Technique}

Amplification technique is a technique used to provide an explanation of a term in the target language. From 47 data obtained, this technique is used in 2 data of translation. as shown in the following table.

Table 7. Amplification Techniques Data

\begin{tabular}{|l|l|l|l|}
\hline NO & \multicolumn{1}{|c|}{ Tsu } & \multicolumn{1}{c|}{ Tsa } & TypesIstilah \\
\hline 1 & の(8) & $\begin{array}{l}\text { ikan cupang } \\
\text { atau Beta } \\
\text { dalam bahasa } \\
\text { Jepang. }\end{array}$ & ekologi-fauna \\
\hline 2 & りの(12) & $\begin{array}{l}\text { Hina-Matsuri } \\
\text { (dolls festival) }\end{array}$ & $\begin{array}{l}\text { organization- } \\
\text { festival }\end{array}$ \\
\hline
\end{tabular}

The use of this technique is intended to provide a deeper explanation regarding a term. On the data this technique is used to translate the cultural terms of thefauna types of the ecology category by combining it with reduction techniques. The word (の) translates as "ikan cupang atau Beta dalam bahasa Jepang". nettaigyo which means "tropical fish" was reduced, then the translator added "in Japanese" to clarify the meaning of the term.
This technique is also used in translating organizational cultural terms related to festival terms which do not have a direct equivalent terms in the target language.

\section{Reduction Technique}

Reduction technique is a technique of reducing or removing words with the aim of condensing the information contained in the source language into the target language. From the 47 data obtained, this technique was used in 6 data translation, such as follow.

Table 8. Reduction Techique Data

\begin{tabular}{|l|l|l|l|}
\hline NO & \multicolumn{1}{|c|}{ Tsu } & \multicolumn{1}{c|}{ Tsa } & \multicolumn{1}{c|}{ Jenis } \\
\hline 1 & $(10)$ & ikan cupang & ekologi-fauna \\
\hline 2 & インドネシアの (6) & kebaya & $\begin{array}{l}\text { material } \\
\text { culture- } \\
\text { clotheses }\end{array}$ \\
\hline 3 & () & bakso & $\begin{array}{l}\text { material } \\
\text { culture-food }\end{array}$ \\
\hline 4 & のったー・(9) & $\begin{array}{l}\text { Mie } \\
\text { Kangkung }\end{array}$ & $\begin{array}{l}\text { material } \\
\text { culture-food }\end{array}$ \\
\hline 5 & claypot & $\begin{array}{l}\text { material } \\
\text { culture-food }\end{array}$ \\
\hline 6 & (の)(21) & "rendang" & $\begin{array}{l}\text { material } \\
\text { culture-food }\end{array}$ \\
\hline
\end{tabular}

This technique is used for cultural terms that come from the target language, especially used in ecology and material culturetypes of cultural terms.

\section{Establish Equivalent Technique}

The establish equivalent technique is a translation technique that uses recognized/common terms or expressions (based on dictionaries or everyday language use). From 47 data obtained, this technique was used in 19 data of translation. such as follows.

Table 9. Establish EquivalentTechnique

\begin{tabular}{|c|c|c|c|}
\hline $\mathrm{NO}$ & $\mathrm{TSu}$ & $\mathrm{TSa}$ & Jenis \\
\hline 1 & インドネシアの (6) & kebaya & $\begin{array}{l}\text { material } \\
\text { culture- } \\
\text { clotheses }\end{array}$ \\
\hline 2 & (12) & batik & $\begin{array}{l}\text { material } \\
\text { culture- } \\
\text { clotheses }\end{array}$ \\
\hline 3 & $-\cdot(7)$ & Mie Ayam & $\begin{array}{l}\text { material } \\
\text { culture-food }\end{array}$ \\
\hline 4 & () & bakso & $\begin{array}{l}\text { material } \\
\text { culture-food }\end{array}$ \\
\hline 5 & のったー・(9) & $\begin{array}{l}\text { Mie } \\
\text { Kangkung }\end{array}$ & $\begin{array}{l}\text { material } \\
\text { culture-food }\end{array}$ \\
\hline 6 & $\begin{array}{l}\text { • (きしめんののきそば } \\
\text { ) (13) }\end{array}$ & $\begin{array}{l}\text { kwetiau } \\
\text { goreng }\end{array}$ & $\begin{array}{l}\text { material } \\
\text { culture-food }\end{array}$ \\
\hline 7 & $-\cdot(14)$ & Bihun Goreng & $\begin{array}{l}\text { material } \\
\text { culture-food }\end{array}$ \\
\hline 8 & りの (19) & mie pangsit & $\begin{array}{l}\text { material } \\
\text { culture-food }\end{array}$ \\
\hline
\end{tabular}




\begin{tabular}{|c|l|l|l|}
\hline 9 & をしたがあります。(5) & $\begin{array}{l}\text { membantu } \\
\text { kelas bahasa } \\
\text { Jepang. }\end{array}$ & $\begin{array}{l}\text { social culture- } \\
\text { work }\end{array}$ \\
\hline 10 & $(2)$ & volunteer & $\begin{array}{l}\text { social culture- } \\
\text { work }\end{array}$ \\
\hline 11 & $-(24)$ & \#diRumahAja & $\begin{array}{l}\text { social culture- } \\
\text { work }\end{array}$ \\
\hline 12 & $-(25)$ & WFH & $\begin{array}{l}\text { social culture- } \\
\text { work }\end{array}$ \\
\hline 13 & の(2) & $\begin{array}{l}\text { ketika duduk } \\
\text { di bangku } \\
\text { kuliah }\end{array}$ & $\begin{array}{l}\text { social culture- } \\
\text { waktu }\end{array}$ \\
\hline 14 & $(3)$ & $\begin{array}{l}\text { masa isolasi } \\
\text { mandiri }\end{array}$ & $\begin{array}{l}\text { social culture- } \\
\text { waktu }\end{array}$ \\
\hline 15 & $(20)$ & $\begin{array}{l}\text { jaman Orde } \\
\text { Baru }\end{array}$ & $\begin{array}{l}\text { social culture- } \\
\text { waktu }\end{array}$ \\
\hline 16 & $(2)$ & Madame & $\begin{array}{l}\text { social culture- } \\
\text { panggilan }\end{array}$ \\
\hline 17 & $(6)$ & $\begin{array}{l}\text { Wakil Ketua } \\
\text { DPR }\end{array}$ & $\begin{array}{l}\text { organization- } \\
\text { posisi dalam } \\
\text { pemerintahan }\end{array}$ \\
\hline 18 & ()$(16)$ & Jabodetabek & $\begin{array}{l}\text { organization- } \\
\text { administras }\end{array}$ \\
\hline 19 & ジヨコ大統領(22) & $\begin{array}{l}\text { Bapak } \\
\text { Presiden Joko } \\
\text { Widodo }\end{array}$ & $\begin{array}{l}\text { organization- } \\
\text { posisi dalam } \\
\text { pemerintahan }\end{array}$ \\
\hline
\end{tabular}

The establish equivalent technique is used in cultural terms, clothesing and food types of material culture, work and time types of social culture, and administrative systems and positions in government types of organizational terms that is exist in the target language.

\section{Pure Borrowing}

Pure borrowing technique is a technique of taking a word or term directly from the source language. From 47 data obtained, this technique is used in 2 data of translation, as follows.

Table 10. Pure Borrowing Technique Data

\begin{tabular}{|c|l|l|l|}
\hline NO & \multicolumn{1}{|c|}{ TSu } & \multicolumn{1}{|c|}{ TSa } & \multicolumn{1}{c|}{ Jenis } \\
\hline 1 & $(4)$ & kimono & $\begin{array}{l}\text { material } \\
\text { culture- } \\
\text { clotheses }\end{array}$ \\
\hline 2 & (=のような $)(18)$ & claypot & $\begin{array}{l}\text { material } \\
\text { culture-food }\end{array}$ \\
\hline
\end{tabular}

This technique is used for clothesing and food types of material culture which does not have a direct equivalent term in the target language.

\section{Transposition Technique}

Transposition technique is a technique to replace the grammatical structure of the source language into the grammatical structure of the target language. From 47 data obtained, this technique was used 5 data translations, as the following.
Table 11. Transposition Tehnique Data

\begin{tabular}{|c|c|c|c|}
\hline $\mathrm{NO}$ & $\mathrm{TSu}$ & $\mathrm{TSa}$ & Jenis \\
\hline 1 & のの(22) & $\begin{array}{l}\text { cucu pertama } \\
\text { saya dan dia } \\
\text { laki-laki }\end{array}$ & $\begin{array}{l}\text { social culture- } \\
\text { kinship }\end{array}$ \\
\hline 2 & (24) & $\begin{array}{l}\text { acara } \\
\text { penyerahan } \\
\text { vaksin secara } \\
\text { daring }\end{array}$ & $\begin{array}{l}\text { social culture- } \\
\text { activities }\end{array}$ \\
\hline 3 & きなげ (19) & $\begin{array}{l}\text { pangsit goreng } \\
\text { yang sangat } \\
\text { besar }\end{array}$ & $\begin{array}{l}\text { material } \\
\text { culture- food }\end{array}$ \\
\hline 4 & (23) & $\begin{array}{l}\text { vaksin buatan } \\
\text { AstraZeneca }\end{array}$ & $\begin{array}{l}\text { material } \\
\text { culture- obat }\end{array}$ \\
\hline 5 & のたち (5) & $\begin{array}{l}\text { kedua anak } \\
\text { saya, } \\
\text { kebetulan dua- } \\
\text { duanya } \\
\text { perempuan. }\end{array}$ & $\begin{array}{l}\text { social culture- } \\
\text { kinship }\end{array}$ \\
\hline
\end{tabular}

This technique is used in translating kinship and activities types of social culture terms, related terms of that do not have a direct equivalent in the target language.

\section{Calque Technique}

Calque technique is a technique of translating a foreign language or phrase into the target language literally, which can be done lexically or structurally. In contrast to literal translation, in this technique the source language structure still appears in the target language or the lexical is still maintained but follows the structure of the target language. From 47 data obtained, this technique was used in 3 data translation, as follow.

Table 12. Calque Technique Data

\begin{tabular}{|c|c|c|c|}
\hline $\mathrm{NO}$ & $\mathrm{TSu}$ & $\mathrm{TSa}$ & Jenis \\
\hline 1 & (12) & boneka Hina & $\begin{array}{l}\text { material } \\
\text { culture-hiasan }\end{array}$ \\
\hline 2 & $(21)$ & $\begin{array}{l}\text { bumbu-bumbu } \\
\text { spicy }\end{array}$ & $\begin{array}{l}\text { material } \\
\text { culture-food }\end{array}$ \\
\hline 3 & (8) & off & $\begin{array}{l}\text { social culture- } \\
\text { work }\end{array}$ \\
\hline
\end{tabular}

This technique is used in translating material culture and social culture category of cultural terms, which do not have a direct equivalent terms in the target language.

\section{Particularization Technique}

The particularization technique is a technique using more specific and concrete terms. From 47 data obtained, this technique was used in the 7 data translation, as follow. 
Table 13. Particularization Technique Data

\begin{tabular}{|c|c|c|c|}
\hline $\mathrm{NO}$ & $\mathrm{TSu}$ & $\mathrm{TSa}$ & Jenis \\
\hline 1 & (5) & $\begin{array}{l}\text { anak kedua } \\
\text { saya }\end{array}$ & $\begin{array}{l}\text { social culture- } \\
\text { panggilan }\end{array}$ \\
\hline 2 & (5) & $\begin{array}{l}\text { teman-teman } \\
\text { semua. }\end{array}$ & $\begin{array}{l}\text { social culture- } \\
\text { panggilan }\end{array}$ \\
\hline 3 & さん(11) & $\begin{array}{l}\text { teman-teman } \\
\text { instagram }\end{array}$ & $\begin{array}{l}\text { social culture- } \\
\text { panggilan }\end{array}$ \\
\hline 4 & (8) & sahabat IG & $\begin{array}{l}\text { social culture- } \\
\text { panggilan }\end{array}$ \\
\hline 5 & (9) & teman semua & $\begin{array}{l}\text { social culture- } \\
\text { panggilan }\end{array}$ \\
\hline 6 & (10) & teman semua & $\begin{array}{l}\text { social culture- } \\
\text { panggilan }\end{array}$ \\
\hline 7 & でー (8) & $\begin{array}{l}\text { Di masa } \\
\text { pandemi dan } \\
\text { di-rumah-aja } \\
\text { (stay-home) }\end{array}$ & $\begin{array}{l}\text { social culture- } \\
\text { waktu }\end{array}$ \\
\hline
\end{tabular}

Particularization techniques are used to translate the vocation terms that exist in the cultural terms of social culture. The interesting thing is the use of different equivalents for the same term using this technique. This can be seen in the translation of the term "Minnasama" which is matched with the words "sahabat IG", "teman semua", and "teman-teman semua" in the translation results.

\section{Generalization Technique Data}

Generalization technique is a technique using a more general term, or the opposite of particularization. From 47 data obtained, this technique is used in 2 data translation, as follow.

Table 14. Generalization Technique Data

\begin{tabular}{|c|l|l|l|}
\hline NO & \multicolumn{1}{|c|}{ TSu } & \multicolumn{1}{c|}{ TSa } & \multicolumn{1}{c|}{ Jenis } \\
\hline 1 & と、も(2) & Mereka & $\begin{array}{l}\text { social culture- } \\
\text { kinship }\end{array}$ \\
\hline 2 & $(5)$ & Yang tertua & $\begin{array}{l}\text { social culture- } \\
\text { kinship }\end{array}$ \\
\hline
\end{tabular}

Generalization techniques are used in translating kinship types of social culture related to terms that do not have a direct equivalent terms in the target language.

\section{Literal Translation Technique Data}

Literal translation technique is a technique of transferring a term word by word with a structure that follows the rules of the target language. From 47 data obtained, this technique is used in 1 data translation as follow.
Table 15. Literal Translation Technique Data

\begin{tabular}{|c|l|l|l|}
\hline NO & \multicolumn{1}{|c|}{ TSu } & \multicolumn{1}{|c|}{ TSa } & \multicolumn{1}{c|}{ Jenis } \\
\hline 1 & の(15) & Yayasan & organization- \\
& & Warga & organization \\
& & Persahabatan & \\
& & (Fukushi & \\
& & Tomo No & \\
& & Kai) & \\
\hline
\end{tabular}

Literal Translation technique is used in translating organizational cultural terms which do not have a direct equivalent terms in the target language.

\subsection{Result}

From the data, we can see that in the cultural diplomacy content on Instagram social media by the translator of the Japan Embassy for Indonesia, there are 42 cultural terms are used, which are divided into 4 major types; ecology, material culture, social culture and organizations, customs and ideas. The most widely used type of term is the social culture type $(47,61 \%)$ which includes terms in work types, description of time types and kinship system types.

To translate these cultural terms, 9 translation techniques are used; Amplification Techniques, Reduction Techniques, Establish Equivalent Techniques, Pure Borrowing, Transposition Techniques, Calque Techniques, Particularization Techniques, Generalization Techniques, and Literal Translation. The 2 most widely used techniques are particularization technique (19.61\%) and establish equivalent (37.26\%). The paticularization technique is used in translating the vocation terms that exist in the cultural terms of social culture. Meanwhile the establish equivalent technique is used in translating kinship and time or activities types of social culture that do not have direct equivalents in the target language.

When translating cultural terms derived from the target language, translators of diplomatic content from the Japanese Embassy mostly use Establish equivalent Techniques, reduction techniques and Particularization Techniques. Meanwhile, when translating cultural terms from the source language which does not have a direct equivalent in the target language, the translator uses the amplification technique, pure borrowing technique, transposition technique, calque technique, generalization technique and literal translation technique.

The determination of the translation technique seems to be carried out according to the needs of the content flow, this can be seen from the use of 2 different translation techniques for the same cultural terms. The 
cultural terms nettai gyo no beta were translated by amplification techniques on data (1) and using data reduction techniques (2). The difference in the use of this technique seems to be done with the consideration that the reader already has a clear frame regarding the cultural term from the translation results in data (1) so that in data (2) the translator decides to use a reduction technique in the translation process which results in a shorter translation.

An interesting finding in the translation of cultural terms in the diplomatic content of the Japanese Embassy is the use of different equivalents for the same term using the same technique. This can be seen in the translation of the term "Minnasama" with the particularization technique, the translator matches this term with the words "friends of IG", "friends of all", and "friends of all" in the translation results.

\section{CONCLUSION}

From the results of the analysis, it can be concluded that in the cultural diplomacy content on Instagram social media by the translator of the Japanese Embassy to Indonesia, 42 cultural terms are used which are divided into 4 major types, namely, ecology, material culture, social culture and organizations, customs and ideas. The most widely used type of term is the term social culture type $(47,61 \%)$. To translate cultural terms derived from the target language, translators of diplomatic content from the Japanese Embassy mostly use the Establish equivalent technique, reduction technique and particularization technique. Meanwhile, when translating cultural terms from the source language which does not have a direct equivalent in the target language, the translator uses the amplification technique, pure borrowing technique, transposition technique, calque technique, generalization technique and literal translation technique. The 2 most used techniques are particularization technique (19.61\%) and establish equivalent $(37.26 \%)$. In the use of these techniques, there are translations that use 1 technique and some use 2 translation techniques for the same cultural terms. And the use of the same technique not on the same terms does not necessarily produce the same equivalent words.

\section{ACKNOWLEDGMENTS}

Finally, this paper finished with Allah's blessing and support from everyone around. This paper is far from perfect, but it is expected that it will be useful to provide evaluation materials and input for related parties, especially related to translation in the diplomatic and digital world. For this reason, constructive thoughtful suggestions are welcomed. Thank you.

\section{REFERENCES}

[1] Fahrurrozi, A. Wicaksono, Sekilas Tentang Bahasa Indonesia: Catatan mengenai kebijakan bahasa, kaidah Pembelajaran Sastra, Penerjemahan dan BIPA, Garudhawaca, 2016.

[2] R. Machali, Pedoman Bagi Penerjemah, Grasindo, 2000.

[3] P. Newmark, A Textbook of Translation, Prantice Hall, 1988.

[4] L. Mollina\& A. Hurtado, Translation Techniques Revisited: A Dinamic and Functionalish Approach, Meta, Vol.47, n.4, 2002 pp.498-472. DOI: https://doi.org/10.7202/008033ar

[5] B. Bugin, Penelitian Kualitatif, Prenada Media Group, 2008.

[6] Huberman, Miles, Analisis Data Kualitatif, Universitas Indonesia, 2014

[7] J. C. Catford, A Linguistic Theory of Translation, Oxford University Press, 1965.

[8] M. Shuttleworth, C. Maira, Dictionary of Translation Studies, St. Jerome Publishing, 1997. 